\title{
Adequação normativa dos planos estaduais de segurança alimentar e nutricional no Brasil
}

\author{
Compliance with guidelines by state plans for \\ food and nutritional security in Brazil
}

\section{Adecuación normativa de los planes estatales de seguridad alimentaria y nutricional en Brasil}

\section{Resumo}

Com o intuito de analisar a adequação dos Planos Estaduais de Segurança Alimentar e Nutricional (PlanSAN) às normas estabelecidas pela Política Nacional de Segurança Alimentar e Nutricional (PNSAN), foi realizada pesquisa descritiva e documental, com coleta de dados entre agosto a outubro de 2016. O acesso aos planos foi realizado na página de Internet da Câmara Interministerial de Segurança Alimentar e Nutricional (CAISAN) ou dos governos estaduais, com coleta de informações complementares no âmbito dos estados. Todos os estados brasileiros aderiram ao Sistema de Segurança Alimentar e Nutricional (SISAN), entretanto menos da metade (13 estados, 48\%) elaborou seus planos, destacando-se alguns aspectos: 5 (38\%) dos PlanSAN tinham vigência correspondente ao plano plurianual do estado; 5 (38\%) dos PlanSAN descreviam os requisitos orçamentários para execução das metas propostas; e 7 (54\%) descreviam mecanismos de monitoramento do plano e apenas 2 (15\%) definiam metodologia para monitoramento de segurança alimentar e nutricional. O menor tempo de existência da CAISAN e de adesão parecem estar relacionados com a inexistência de PlanSAN. Ainda que a maioria dos estados com planos atendam algumas normativas estabelecidas pela PNSAN, esses instrumentos tornam-se frágeis e pouco exequíveis quando não possuem vinculação orçamentária para suas metas. Pelo fato de a PNSAN ser estruturalmente intersetorial, a construção dos planos depende de um trabalho coletivo das diversas secretarias de governo. Reforça-se que os itens analisados foram todos normativos, o que implica necessidade do estabelecimento de mecanismos que garantam a sua adequada execução.

Segurança Alimentar e Nutricional; Programas de Nutrição; Avaliação de Programas e Projetos de Saúde

\author{
Mick Lennon Machado 1 \\ Cristine Garcia Gabriel 1 \\ Claudia Soar 2 \\ Gisele Rockenbach Mamed 2 \\ Patrícia Maria de Oliveira Machado 3 \\ Josimari Telino de Lacerda 3 \\ Milena Corrêa Martins 4 \\ Maria Cristina Marcon 3
}

doi: 10.1590/0102-311X00206716

\author{
Correspondência \\ C. G. Gabriel \\ Programa de Pós-graduação em Nutrição, Universidade Federal \\ de Santa Catarina. \\ Centro de Ciências da Saúde, Campus Universitário Trindade, \\ Florianópolis, SC 88040-970, Brasil. \\ cristine.gabriel@ufsc.br

\footnotetext{
1 Programa de Pós-graduação em Nutrição, Universidade Federal de Santa Catarina, Florianópolis, Brasil. 2 Departamento de Nutrição, Universidade Federal de Santa Catarina, Florianópolis, Brasil.

3 Programa de Pós-graduação em Saúde Coletiva, Universidade Federal de Santa Catarina, Florianópolis, Brasil.

4 Teia de Articulação pelo Fortalecimento da Segurança Alimentar e Nutricional, Universidade Federal de Santa Catarina, Florianópolis, Brasil.
} 


\section{Introdução}

O Sistema Nacional de Segurança Alimentar e Nutricional (SISAN) foi instituído no Brasil em 2006 por meio da Lei Orgânica de Segurança Alimentar e Nutricional (LOSAN). É considerado um marco institucional no campo das políticas de alimentação e nutrição por propor a alimentação adequada como direito de todos e prioridade política de governo ${ }^{1}$. A análise dos programas de combate à fome no Brasil no período de 1930 a 2003 aponta importantes mudanças, sobretudo depois da metade dos anos 19802 e da criação do programa Fome Zero, em 2003.

Entretanto, somente a partir de 2010, a alimentação passou a ser garantida como direito básico na Constituição Federal 3. Ainda nesse ano, o Decreto no 7.272/2010 4 instituiu a Política Nacional de Segurança Alimentar e Nutricional (PNSAN), estabelecendo quatro elementos fundamentais para estruturação do SISAN: os Conselhos de Segurança Alimentar e Nutricional (CONSEA), as Câmaras de Gestão Intersetorial de Segurança Alimentar e Nutricional (CAISAN), as Conferências de Segurança Alimentar e Nutricional (CSAN) e os Planos Estaduais de Segurança Alimentar e Nutricional (PlanSAN), em um modelo que deve ser replicado nas três esferas de governo por meio da adesão dos níveis estadual e municipal.

No Brasil, assume-se que segurança alimentar e nutricional é a "realização do direito de todos ao acesso regular e permanente a alimentos de qualidade, em quantidade suficiente, sem comprometer o acesso a outras necessidades essenciais, tendo como base práticas alimentares promotoras de saúde que respeitem a diversidade cultural e que sejam ambiental, cultural, econômica e socialmente sustentáveis" 1 . O conceito incorpora dimensões relativas à quantidade e qualidade de alimentos, acesso, práticas sustentáveis de produção agrícola, cidadania e direitos humanos, a depender da situação epidemiológica e contexto socioeconômico de cada local. Deve ser traduzido em ações nos PlanSAN, expressando os compromissos pactuados pela gestão pública com a sociedade civil para ampliar e efetivar ações de garantia da segurança alimentar e nutricional, pautadas em construções participativas e intersetoriais fruto das diferentes instâncias $1,4,5,6$.

Ressalta-se a intersetorialidade como pilar fundamental da PNSAN e consolidação do SISAN, e, neste sentido, um enorme desafio para sua estruturação. Os diferentes mecanismos institucionais de articulação ainda possuem longo caminho para estabelecer consensos estratégicos em prol da política pública de segurança alimentar e nutricional. Além da necessidade do planejamento conjunto entre diferentes setores governamentais que possuem histórico de um complexo processo decisório, a PNSAN possui o desafio de articular os três níveis de gestão interfederativa 5 .

Nessa perspectiva, os PlanSAN constituem-se instrumento central do SISAN por serem a principal ferramenta de planejamento, gestão e execução da PNSAN nas diferentes esferas de governo 4. Considerando que os planos têm o intuito de refletir a situação de segurança alimentar e nutricional, além de aspirar à integração e ao fortalecimento de distintas políticas e programas para garantia da segurança alimentar e nutricional, pressupõe-se que a adequação desse instrumento às normas e critérios pré-estabelecidos contribua para a execução local da PNSAN. Tendo em vista a literatura ainda incipiente acerca do SISAN e seus componentes, ressalta-se a necessidade de estudos que subsidiem estratégias para a qualificação dos planos enquanto ferramenta de gestão da PNSAN.

Dadas as características inovadoras e desafiadoras para a consolidação do SISAN, bem como as inúmeras peculiaridades inerentes às regiões do Brasil, este artigo tem como propósito analisar a adequação dos PlanSAN às normas estabelecidas pela PNSAN.

\section{Métodos}

Trata-se de uma pesquisa descritiva e documental, com coleta de dados realizada entre os meses de agosto a outubro de 2016.

Para análise dos PlanSAN, foram observados os itens normativos que orientam sua construção, dispostos no Capítulo VII do Decreto no 7.272/2010 4, intitulado Da Operacionalização da Política Nacional de Segurança Alimentar e Nutricional. Utilizaram-se como referência os parâmetros estabelecidos nos Art. 18, 19, 20 e 21 que dispõem que os planos devem ser elaborados tendo em conta as propostas das CSAN, priorizadas e sistematizadas pelo CONSEA, sendo construídos intersetorialmente pela 
CAISAN. Devem conter a análise de situação de segurança alimentar e nutricional; ter vigência correspondente ao plano plurianual (PPA); consolidar programas e ações, indicando prioridades, metas e requisitos orçamentários; definir responsabilidades dos órgãos e entidades; atender a especificidades dos diversos grupos em situação de insegurança alimentar e nutricional; e definir mecanismos de monitoramento e avaliação da situação de segurança alimentar e nutricional e do próprio plano. Os planos devem ser elaborados respeitando as oito diretrizes da PNSAN que traduzem o conceito de segurança alimentar e nutricional adotado pelo país. Além disso, devem ser revisados a cada dois anos 4 . A Figura 1 representa o modelo teórico proposto para os PlanSAN, estando em consonância com os pressupostos estabelecidos pela Lei no 11.346/2006 1 e pelo Decreto no 7.272/2010 4 .

Os estados com PlanSAN publicados a partir da regulamentação do SISAN foram identificados por meio de contato com a CAISAN Nacional. O acesso aos planos foi realizado na página de Internet da própria CAISAN (http://mds.gov.br/caisan-mds) e dos governos estaduais ou pelo contato com as CAISAN estaduais. Procedeu-se à leitura detalhada e se verificou se os planos: eram quadrienais; tinham vigência correspondente ao PPA do estado; continham análise de situação de segurança alimentar e nutricional, considerando as sete dimensões definidas no Art. 21 do Decreto no 7.272/2010 4; explicitavam os responsáveis pelas metas; indicavam metas prioritárias especificadas conforme as oito diretrizes de segurança alimentar e nutricional previstas no Art. 3 do Decreto no 7.272/2010 4;

\section{Figura 1}

Modelo teórico dos Planos Estaduais de Segurança Alimentar e Nutricional (PlanSAN).

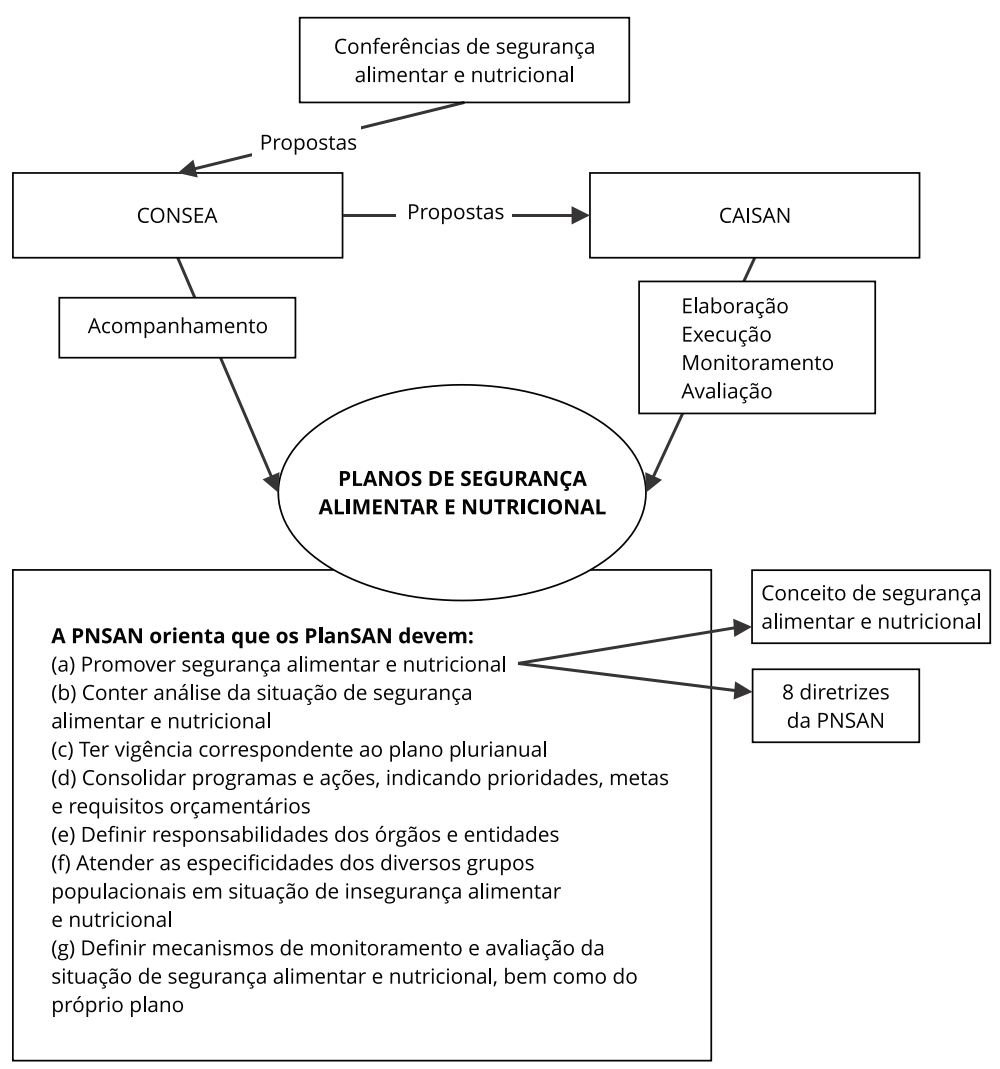

CAISAN: Câmara Estadual Intersetorial de Segurança Alimentar e Nutricional; CONSEA: Conselho Estadual de Segurança Alimentar e Nutricional; PNSAN: Política Nacional de Segurança Alimentar e Nutricional. 
apresentavam requisitos orçamentários para as metas; definiam processos e responsáveis pelo monitoramento e avaliação do plano; e definiam processos de monitoramento e avaliação de segurança alimentar e nutricional.

Também foram coletadas informações complementares no âmbito dos estados, a saber: ano de criação do CONSEA e da CAISAN; ano de adesão ao SISAN; número e campo de atuação das secretarias participantes da CAISAN; secretaria de coordenação da PlanSAN; existência de recursos financeiros estaduais para funcionamento da CAISAN; e existência de recursos financeiros para funcionamento da PlanSAN. As variáveis foram coletadas junto à Pesquisa de Informações Básicas Estaduais - Estadic (Instituto Brasileiro de Geografia e Estatística - IBGE. http://www.ibge.com.br/ home/estatistica/economia/estadic/default.shtm, acessado em 01/Nov/2016), com exceção do ano de criação da CAISAN, obtido por meio de solicitação à CAISAN Nacional; do ano de adesão ao SISAN, coletado no Diário Oficial da União 7; e das informações a respeito das CAISAN e coordenação da PNSAN, coletadas nas legislações estaduais.

Realizou-se análise exploratória das variáveis investigadas e, com base nos parâmetros do Decreto no 7.272/2010 4, os planos foram verificados quanto à sua adequação a aspectos normativos dispostos na PNSAN. Os anos de criação do CONSEA, da CAISAN e de adesão ao SISAN foram tratados como variáveis qualitativas. Para as duas primeiras. foram utilizadas as seguintes categorias: antes do ano de criação do Fome Zero (<2003); a partir do ano de criação do Fome Zero e antes do ano de publicação da LOSAN (2003-2005); a partir do ano de publicação da LOSAN e antes do ano de regulamentação do SISAN (2006-2009); e a partir do ano de regulamentação do SISAN ( $\geq 2010)$. O ano de adesão ao SISAN foi descrito pelas seguintes categorias: a partir do ano de regulamentação do SISAN até o final do ano seguinte (2011) e de 2012 em diante. O campo de atuação das secretarias foi analisado com base em 13 categorias: desenvolvimento econômico e apoio à indústria e ao comércio; desenvolvimento rural, agricultura, pecuária, pesca e abastecimento; direitos humanos, cidadania, assistência social e políticas afirmativas; educação, esporte, cultura, ciência e tecnologia; governo e casa civil; justiça e segurança pública; meio ambiente, recursos hídricos e saneamento; planejamento, gestão e fazenda; saúde; segurança alimentar e nutricional; transporte, infraestrutura, logística e cidades; turismo; outros. As seguintes variáveis foram analisadas dicotomicamente: existência de recursos financeiros para CAISAN e para política de segurança alimentar e nutricional; indicação de metas prioritárias; definição de processos e de responsáveis para monitoramento do plano; e definição de processos para monitoramento da situação de segurança alimentar e nutricional. As variáveis: indicação de responsáveis pelas metas e dos requisitos orçamentários foram categorizadas em sim, não e parcial, sendo a última categoria utilizada quando nem todas as metas apresentavam as informações.

As informações coletadas foram sistematizadas em planilha do Excel 2016 (Microsoft Corp. Estados Unidos), e os dados foram apresentados na forma de distribuição de frequência simples para as variáveis qualitativas e pela média ou mediana para as quantitativas, respeitando a distribuição normal das variáveis.

O presente artigo faz parte de um projeto de extensão, financiado pelo Edital de Chamamento Público MDS/SESAN no 01/2013 que contemplou projetos voltados ao fortalecimento do SISAN nos estados e seus respectivos municípios.

\section{Resultados}

Todos os estados brasileiros e o Distrito Federal possuíam CONSEA, com período de criação entre 1999 e 2011. Três (11\%) conselhos foram instalados antes da criação do Fome Zero, em 2003; 17 (63\%) a partir do ano de criação do Fome Zero e antes do ano de publicação da LOSAN, em 2006; 4 (15\%) a partir do ano de publicação da LOSAN e antes do ano de regulamentação do SISAN, em 2010; e 3 (11\%) pela regulamentação do SISAN. Os primeiros estados a criarem CONSEA foram Mato Grosso do Sul e Minas Gerais, ambos em 1999. Da mesma forma, todos os estados e o Distrito Federal possuíam CAISAN, com data de criação entre 2006 a 2014. Minas Gerais também foi o primeiro estado a criar CAISAN no país, estando dentre os cinco (19\%) estados que implementaram suas câmaras entre 2006 e 2009. Os 22 estados (81\%) restantes criaram suas câmaras a partir do ano de regulamentação do SISAN. Todos os estados já aderiram ao Sistema, em processo iniciado em 2011, com 22 (81\%) estados. 
Os cinco estados restantes aderiram ao SISAN entre 2012 e 2014, sendo Mato Grosso o último estado a realizar a adesão (Tabela 1).

Treze estados, incluindo o Distrito Federal (48\%), elaboraram PlanSAN após regulamentação do SISAN, sendo os primeiros publicados em 2012 (Ceará, Minas Gerais, Pará e Paraná) e o último em 2016 (Paraíba). Quanto às regiões brasileiras, destaca-se a Sul como a única onde todos os estados possuíam planos publicados. Cinco (55\%) estados do Nordeste possuíam PlanSAN; 2 (50\%) do Centrooeste; 2 (29\%) do Norte; e 1 (25\%) do Sudeste (Tabela 1).

Dentre os estados que publicaram planos, o tempo médio do CONSEA era de 12,7 anos, da CAISAN de 7,1 anos e todos realizaram a adesão em 2011. Já entre os estados que ainda não publicaram PlanSAN, o tempo médio do CONSEA era de 12,8 anos, da CAISAN de 5,4 anos e 5 (36\%) aderiram ao SISAN tardiamente, entre 2012 e 2014.

As CAISAN estaduais eram constituídas por uma média de 10,7 secretarias, com variação entre 5 em Sergipe e 22 na Paraíba. Todas possuíam secretarias ou órgãos de governo com campos de atuação em desenvolvimento rural, agricultura, pecuária, pesca e abastecimento; direitos humanos, cidadania, assistência social e políticas afirmativas; educação, esporte, cultura, ciência e tecnologia; e saúde. Ainda, 22 (88\%) das CAISAN contavam com secretarias ou órgãos relacionados a meio ambiente, recursos hídricos e saneamento. A coordenação da PlanSAN estava alocada em secretarias de direitos humanos, cidadania, assistência social e políticas afirmativas em 21 (78\%) dos estados, enquanto 3 (11\%) vincularam a coordenação ao desenvolvimento rural, agricultura, pecuária, pesca e abastecimento; 2 (7\%), à casa civil ou governo; e 1 (4\%), à secretaria específica de segurança alimentar e nutricional (Piauí) (dados não apresentados em tabela).

Apenas 11 (41\%) estados afirmaram dispor de recursos orçamentários estaduais para apoiar o funcionamento da CAISAN. Dentre os 13 estados com planos, 6 (46\%) referiram contar com esses recursos versus 5 (36\%) dos estados sem planos. Havia recursos previstos para o funcionamento da PNSAN em 24 (89\%) dos estados, excetuando-se Mato Grosso, Mato Grosso do Sul e Amazonas (Tabela 1).

$\mathrm{Na}$ Tabela 2, observa-se que dentre os estados com planos, 5 (38\%) apresentavam vigência quadrienal, 5 (38\%), vigência inferior a quatro anos e 3 (24\%), vigência superior. O plano de Tocantins apresentava vigência de 1 ano, enquanto o plano de Santa Catarina possuía vigência de 6 anos. Cinco (38\%) dos planos tinham vigência correspondente ao PPA do estado. Em $6(46 \%)$ estados, os planos apresentavam período de vigência inferior ao PPA, e em 2 (16\%) a vigência era superior. Em 9 (69\%) dos planos, a análise da situação estadual de segurança alimentar e nutricional foi realizada tendo em conta todas as sete dimensões de monitoramento e avaliação (Tabela 2). Constatou-se que os planos do Maranhão, Minas Gerais e Paraná, embora contivessem a análise da situação, não abordavam a dimensão referente aos programas e ações relacionadas à segurança alimentar e nutricional. Já Tocantins foi o único estado com plano sem análise estadual da situação de segurança alimentar e nutricional (dados não apresentados em tabela).

A mediana do número de metas dos PlanSAN foi igual a 102; o plano do Maranhão apresentou o menor número de metas (13) e o plano do Rio Grande do Sul o maior (297) (Tabela 2). Somente 2 (15\%) dos planos indicavam metas especificadas pelas oito diretrizes de segurança alimentar e nutricional, enquanto $9(70 \%)$ especificavam parte das diretrizes. Nesses 9 planos, não havia metas específicas para a diretriz que trata do apoio a iniciativas de promoção da soberania alimentar, segurança alimentar e nutricional e direito humano à alimentação adequada (DHAA). O plano do Maranhão também não apresentava metas especificadas para a instituição de processos permanentes de educação alimentar e nutricional, pesquisa e formação nas áreas de segurança alimentar e nutricional e do DHAA, nem para a diretriz referente à promoção do acesso universal à água de qualidade e em quantidade suficiente. Já os planos do Pará e Tocantins também não especificaram metas para a diretriz de monitoramento da realização do DHAA. Os planos da Bahia e Minas Gerais (15\%) continham suas metas especificadas por diretrizes diferentes daquelas descritas na PNSAN (dados não apresentados em tabela).

Com exceção do plano de Minas Gerais, os outros 12 (92\%) planos explicitavam as responsabilidades dos órgãos e entidades para todas as metas propostas. Onze (85\%) dos planos indicavam as metas prioritárias, mas os planos de Tocantins e Paraná não cumpriam com essa normativa. Cinco (38\%) planos (Bahia, Maranhão, Pernambuco, Minas Gerais e Paraná) descreviam os requisitos orçamentários para execução de todas as metas propostas, 4 (31\%) apresentavam os requisitos para parte das metas e 4 (31\%) não os expunham para qualquer meta (Tabela 2). 
Tabela 1

Panorama da estruturação do Sistema Nacional de Segurança Alimentar e Nutricional (SISAN) nos estados e no Distrito Federal. Brasil, 2016.

\begin{tabular}{|c|c|c|c|c|c|c|c|}
\hline Estados & $\begin{array}{c}\text { Ano de } \\
\text { criação do } \\
\text { CONSEA }\end{array}$ & $\begin{array}{c}\text { Ano de } \\
\text { criação do } \\
\text { CAISAN }\end{array}$ & $\begin{array}{c}\text { Ano de } \\
\text { adesão ao } \\
\text { SISAN }\end{array}$ & $\begin{array}{c}\text { Ano de } \\
\text { publicação } \\
\text { do PlanSAN }\end{array}$ & $\begin{array}{l}\text { Secretarias } \\
\text { do CAISAN }\end{array}$ & $\begin{array}{c}\text { Existência } \\
\text { de recursos } \\
\text { (CAISAN) }\end{array}$ & $\begin{array}{c}\text { Existência } \\
\text { de recursos } \\
\text { (PNSAN) }\end{array}$ \\
\hline \multicolumn{8}{|l|}{ Centro-oeste } \\
\hline Distrito Federal & 2008 & 2011 & 2011 & 2013 & 12 & Sim & Sim \\
\hline Goiás & 2004 & 2013 & 2013 & - & 9 & Sim & Sim \\
\hline Mato Grosso & 2003 & 2014 & 2014 & - & 10 & Não & Não \\
\hline Mato Grosso do Sul & 1999 & 2011 & 2011 & 2015 & 6 & Sim & Não \\
\hline \multicolumn{8}{|l|}{ Nordeste } \\
\hline Alagoas & 2000 & 2011 & 2011 & - & 9 & Não & Sim \\
\hline Bahia & 2003 & 2008 & 2011 & 2015 & 11 & Sim & Sim \\
\hline Ceará & 2003 & 2011 & 2011 & 2012 & 12 & Não & Sim \\
\hline Maranhão & 2006 & 2011 & 2011 & 2014 & 9 & Sim & Sim \\
\hline Paraíba & 2003 & 2012 & 2011 & 2016 & 22 & Não & Sim \\
\hline Pernambuco & 2003 & 2008 & 2011 & 2013 & 13 & Não & Sim \\
\hline Piauí & 2003 & 2009 & 2011 & - & 14 & Não & Sim \\
\hline Rio Grande do Norte & 2003 & 2011 & 2012 & - & 7 & Não & Sim \\
\hline Sergipe & 2003 & 2013 & 2011 & - & 5 & Não & Sim \\
\hline \multicolumn{8}{|l|}{ Norte } \\
\hline Acre & 2004 & 2011 & 2011 & - & 11 & Não & Sim \\
\hline Amapá & 2006 & 2011 & 2011 & - & 8 & Sim & Sim \\
\hline Amazonas & 2004 & 2012 & 2012 & - & 12 & Sim & Não \\
\hline Pará & 2011 & 2011 & 2011 & 2012 & 7 & Sim & Sim \\
\hline Rondônia & 2008 & 2011 & 2011 & - & 9 & Não & Sim \\
\hline Roraima & 2003 & 2011 & 2011 & - & 10 & Sim & Sim \\
\hline Tocantins & 2010 & 2010 & 2011 & 2014 & 7 & Não & Sim \\
\hline \multicolumn{8}{|l|}{ Sudeste } \\
\hline Espírito Santo & 2011 & 2011 & 2011 & - & 13 & Sim & Sim \\
\hline Minas Gerais & 1999 & 2006 & 2011 & 2012 & 13 & Sim & Sim \\
\hline Rio de Janeiro & 2003 & 2010 & 2011 & - & 10 & Não & Sim \\
\hline São Paulo & 2003 & 2013 & 2013 & - & 20 & Sim & Sim \\
\hline \multicolumn{8}{|l|}{ Sul } \\
\hline Paraná & 2003 & 2010 & 2011 & 2012 & 10 & Não & Sim \\
\hline Rio Grande do Sul & 2003 & 2011 & 2011 & 2014 & 12 & Não & $\operatorname{Sim}$ \\
\hline Santa Catarina & 2004 & 2009 & 2011 & 2014 & 9 & Não & Sim \\
\hline
\end{tabular}

CAISAN: Câmara Estadual Intersetorial de Segurança Alimentar e Nutricional; CONSEA: Conselho Estadual de Segurança Alimentar e Nutricional;

PlanSAN: Planos Estaduais de Segurança Alimentar e Nutricional; PNSAN: Política Nacional de Segurança Alimentar e Nutricional.

Em 7 (54\%) planos, havia descrição da metodologia para monitoramento da execução do plano e, em 9 (69\%), foram descritos os responsáveis por realizar tal monitoramento. Entretanto, somente 2 (15\%) planos apresentavam metodologia para monitoramento da situação de segurança alimentar e nutricional (Tabela 3).

\section{Discussão}

Os dados apresentados constituem uma representação inicial do processo de elaboração dos PlanSAN. Dentre as possíveis limitações do estudo, destaca-se sua natureza descritiva e restringir-se 
Tabela 2

Caracterização dos Planos Estaduais de Segurança Alimentar e Nutricional (PlanSAN) dos estados e do Distrito Federal quanto à vigência, análise da situação de segurança alimentar e nutricional e metas. Brasil, 2016.

\begin{tabular}{|c|c|c|c|c|c|c|c|c|}
\hline Estados & $\begin{array}{l}\text { Vigência do } \\
\text { PlanSAN }\end{array}$ & $\begin{array}{l}\text { Vigência } \\
\text { do PPA }\end{array}$ & $\begin{array}{c}\text { Dimensões } \\
\text { na } \\
\text { análise da } \\
\text { situação de } \\
\text { segurança } \\
\text { alimentar e } \\
\text { nutricional }\end{array}$ & Metas & $\begin{array}{c}\text { Diretrizes nas } \\
\text { metas }\end{array}$ & $\begin{array}{l}\text { Responsável } \\
\text { pelas metas }\end{array}$ & $\begin{array}{c}\text { Metas } \\
\text { prioritárias }\end{array}$ & $\begin{array}{c}\text { Requisito } \\
\text { orçamentário }\end{array}$ \\
\hline \multicolumn{9}{|l|}{ Centro-oeste } \\
\hline Distrito Federal & $2013-2016$ & $2012-2015$ & 7 & 144 & 7 & Sim & Sim & Parcial \\
\hline Mato Grosso do Sul & $2015-2016$ & $2012-2015$ & 7 & 102 & 7 & Sim & Sim & Não \\
\hline \multicolumn{9}{|l|}{ Nordeste } \\
\hline Bahia & 2015-2019 & 2016-2019 & 7 & 78 & 0 * & Sim & Sim & Sim \\
\hline Ceará & 2012-2015 & $2012-2015$ & 7 & 105 & 8 & Sim & Sim & Parcial \\
\hline Maranhão & 2014-2015 & $2012-2015$ & 6 & 13 & 5 & Sim & Sim & Sim \\
\hline Paraíba & 2016-2019 & 2016-2019 & 7 & 237 & 7 & Sim & $\operatorname{Sim}$ & Parcial \\
\hline Pernambuco & 2013-2015 & $2012-2015$ & 7 & 214 & 7 & Sim & Sim & Sim \\
\hline \multicolumn{9}{|l|}{ Norte } \\
\hline Pará & $2012-2015$ & $2012-2015$ & 7 & 56 & 6 & Sim & Sim & Não \\
\hline Tocantins & 2014 & $2012-2015$ & 0 & 69 & 6 & Sim & Não & Não \\
\hline \multicolumn{9}{|l|}{ Sudeste } \\
\hline Minas Gerais & $2012-2015$ & $2012-2015$ & 6 & 65 & 0 * & Não & $\operatorname{Sim}$ & Sim \\
\hline \multicolumn{9}{|l|}{ Sul } \\
\hline Paraná & $2012-2015$ & $2012-2015$ & 6 & 67 & 7 & Sim & Não & Sim \\
\hline Rio Grande do Sul & 2014-2015 & $2012-2015$ & 7 & 297 & 8 & Sim & Sim & Parcial \\
\hline Santa Catarina & 2014-2019 & $2016-2019$ & 7 & 168 & 7 & Sim & Sim & Não \\
\hline
\end{tabular}

PPA: plano plurianual.

* Seguem diretrizes próprias adequadas à realidade local.

a análises documentais, não contemplando neste momento todos os componentes do SISAN. Todavia, reforça-se que o foco foram os PlanSAN, pela sua centralidade no sistema, investigando-se todos os estados brasileiros. Buscando avançar nas discussões e ultrapassar análises superficiais e/ou insuficientes, complementou-se a avaliação dos itens normativos dos planos com outros elementos, os quais possibilitaram a obtenção de um panorama acerca do processo de estruturação do SISAN nos estados brasileiros e suas possíveis relações com a elaboração dos PlanSAN. Esses esforços, aliados à escassez de estudos relacionados à temática na literatura investigada, conferem ao presente estudo a possibilidade de contribuir para a qualificação das discussões e ações em torno da PNSAN.

Entende-se que a discussão em torno da PNSAN precisa estar contextualizada. Torna-se essencial destacar que, historicamente, o Brasil conta com políticas e programas setoriais que objetivam responder por elementos de segurança alimentar e nutricional 2,5. As iniciativas brasileiras, além de responderem por uma reivindicação interna de diversos movimentos sociais, também são fruto de um contexto internacional. Já foram identificadas políticas públicas de segurança alimentar e nutricional em 123 países, especialmente naqueles menos desenvolvidos 8 . Percebe-se que em países onde a fome e a miséria caracterizam-se como principal problema, o conceito e as políticas de segurança alimentar e nutricional estão mais associados à dimensão do acesso a quantidades suficientes de alimentos 9,10,11,12. Nos países onde a fome foi superada, o conceito e as políticas são direcionados para os aspectos qualitativos da alimentação 13,14,15,16,17. Já em países em transição epidemiológica, como o Brasil, assumem-se conceitos e políticas que envolvem ambas as dimensões 2,18,19,20.

A despeito disto, somente em 2006, o Brasil consegue avançar para a construção de uma política pública intersetorial de segurança alimentar e nutricional, que possui como eixo principal "associar a 


\section{Tabela 3}

Caracterização dos Planos Estaduais de Segurança Alimentar e Nutricional (PlanSAN) dos estados e do Distrito Federal quanto ao processo de monitoramento e avaliação. Brasil, 2016.

\begin{tabular}{|c|c|c|c|}
\hline Estados & $\begin{array}{c}\text { Metodologia de } \\
\text { monitoramento do PlanSAN }\end{array}$ & $\begin{array}{c}\text { Responsáveis pelo } \\
\text { monitoramento do PlanSAN }\end{array}$ & $\begin{array}{c}\text { Metodologia de monitoramento } \\
\text { de segurança alimentar e } \\
\text { nutricional }\end{array}$ \\
\hline \multicolumn{4}{|l|}{ Centro-oeste } \\
\hline Distrito Federal & Sim & Sim & Não \\
\hline Mato Grosso do Sul & Sim & Sim & Não \\
\hline \multicolumn{4}{|l|}{ Nordeste } \\
\hline Bahia & Sim & Sim & Sim \\
\hline Ceará & Não & Sim & Não \\
\hline Maranhão & Sim & Sim & Não \\
\hline Paraíba & Não & Não & Não \\
\hline Pernambuco & Não & Sim & Não \\
\hline \multicolumn{4}{|l|}{ Norte } \\
\hline Pará & Sim & Não & Não \\
\hline Tocantins & Não & Sim & Não \\
\hline \multicolumn{4}{|l|}{ Sudeste } \\
\hline Minas Gerais & Sim & Sim & Não \\
\hline \multicolumn{4}{|l|}{ Sul } \\
\hline Paraná & Não & Não & Não \\
\hline Rio Grande do Sul & Não & Sim & Não \\
\hline Santa Catarina & Sim & Não & Sim \\
\hline
\end{tabular}

segurança alimentar a estratégias permanentes de desenvolvimento econômico e social com crescente equidade e inclusão” 21 (p. 17). Apesar de um contexto internacional de inúmeras experiências para promoção de segurança alimentar e nutricional, e de um histórico de luta e construção de ações para garantia do DHAA no Brasil, o processo de elaboração dos PlanSAN não é uniforme nos estados, refletindo as distintas trajetórias em relação à PNSAN.

Mesmo todos os estados tendo aderido ao Sistema após a regulamentação da LOSAN e criação da PNSAN 1,4, até o momento menos da metade elaborou seus PlanSAN. Dentre as variáveis de estruturação do SISAN analisadas, o menor tempo de existência da CAISAN e de adesão parecem estar relacionados com a inexistência de PlanSAN.

A existência de três CONSEA estaduais anteriores ao lançamento do Fome Zero e construção da PNSAN pode ter sido motivada pela instituição do CONSEA nacional em 1993 2,18. Contudo, naquele momento, mesmo com o lançamento de um plano nacional de combate à fome e à miséria, o conselho nacional teve duração curta, não chegando à década seguinte 2,18. Diante desse contexto, não foram encontradas novas iniciativas de conselhos estaduais até a criação do Fome Zero, em 200321. Nesse mesmo ano, ocorreu a recriação do CONSEA nacional e doze estados brasileiros instituíram seus conselhos. Os demais foram instituindo nos anos seguintes, alguns após a regulamentação da LOSAN 1.

Quanto às CAISAN estaduais, verificou-se que a maioria dos estados a implementaram após da instituição da PNSAN 4, que definiu as atribuições dos órgãos e entidades dos estados e do Distrito Federal na gestão do SISAN. Porém, destaca-se a iniciativa de quatro estados de criarem suas câmaras logo após a regulamentação da LOSAN, que estabeleceu o modo de funcionamento do SISAN. Minas Gerais destaca-se como o primeiro estado brasileiro a ter CAISAN, sendo também um dos primeiros estados a publicar seu PlanSAN. Em contraposição, o Estado do Mato Grosso criou sua CAISAN quase dez anos após a LOSAN 1, não tendo ainda PlanSAN. 
A adesão dos estados ao SISAN ocorreu majoritariamente no ano seguinte à implantação da PNSAN, com diferença de quatro anos no período de adesão entre todos os estados. Embora o prazo legal para publicação dos PlanSAN seja de um ano após a adesão formal do ente federado ao SISAN 4, menos da metade dos estados publicou seus planos, e a maioria deles os publicou em período superior ao estipulado pela PNSAN 4. O Estado da Paraíba, por exemplo, aderiu ao SISAN em 2011, mas divulgou seu PlanSAN somente em 2016. Casos como esses suscitam questionamentos e investigações acerca dos fatores que diferenciam os estados na implantação e consolidação de suas políticas públicas. Há que se considerar que as diferenças encontradas nos PlanSAN dos estados podem estar relacionadas a diversos fatores inerentes ao sistema federativo brasileiro, marcado por "iniquidades e diversidades sociais, politicas, institucionais e de gestão, além das redes de relações de poder local" 22 (p. 87). Todavia, questões próprias da PNSAN auxiliam a compreender os resultados encontrados nesta pesquisa.

Pelo fato de a PNSAN ser estruturalmente intersetorial, a construção dos planos depende de um trabalho coletivo das diversas secretarias de governo com base nas CAISAN. E diante de uma histórica organização setorial da gestão pública, a promoção da intersetorialidade ainda é desafiadora, constituindo um dos pontos de maior dificuldade para a construção do Plano Distrital de Segurança Alimentar e Nutricional em função de aspectos relacionados à intercomunicação, prazos, cumprimento de agenda e cooperação entre as esferas do governo ${ }^{23}$. A composição das CAISAN demonstra que os estados compreendem que a garantia do DHAA e a promoção de segurança alimentar e nutricional está relacionada com os campos de atuação dos direitos humanos, assistência social, agricultura, educação, saúde e meio ambiente. Tais campos respondem pelas diretrizes da PNSAN 4 e, portanto, as CAISAN estaduais apresentam potencial para construir planos capazes de atender ao conceito de segurança alimentar e nutricional. Entretanto, retomando-se esse conceito e a essencialidade das ações intersetoriais para seu pleno alcance, pressupõe-se que a existência de câmaras com número pequeno de secretarias pode limitar o planejamento e execução de ações e estratégias efetivas.

Ainda, a PNSAN 4 orienta que as estruturas do SISAN estejam alocadas acima das estruturas setoriais dos ministérios e secretarias, como forma de garantir ações intersetoriais. Contudo, constatou-se que, em nível estadual, a Política de Segurança Alimentar e Nutricional é coordenada essencialmente por secretarias vinculadas ao campo de atuação relacionado aos direitos humanos, cidadania, assistência social e políticas afirmativas. Com isso, pode-se limitar a potencialidade intersetorial da PNSAN e, consequentemente, dos próprios planos. Somado a isso, preocupa também o fato de menos da metade dos estados referir disponibilidade de recursos financeiros para as CAISAN, reforçando o enorme desafio do Brasil em instituir e consolidar esse Sistema, sobretudo ao se considerar os interesses econômicos inerentes a uma política que pretende garantir o bem-estar social acima das necessidades de acumulação de capital 5 .

Ao analisar as metas, verificou-se que os planos respeitavam boa parte das diretrizes de segurança alimentar e nutricional. Porém, somente Ceará e Rio Grande do Sul descreveram metas para a diretriz relacionada à soberania alimentar. O conceito de soberania alimentar está presente nas discussões internacionais sobre segurança alimentar e nutricional desde a sua origem, ainda no período seguinte à I Guerra Mundial 24. No entanto, é um dos conceitos mais recentes a ser introduzido no conceito de segurança alimentar e nutricional adotado pelo Brasil 25. A dificuldade dos estados em construir metas relacionadas a essa diretriz pode ser justificada pela compreensão da soberania como elemento das relações internacionais e, portanto, condizente apenas à realidade do PNSAN 4. A promoção da soberania alimentar, no entanto, também passa por iniciativas locais de desenvolvimento de "políticas públicas que estimulem e organizem a produção de alimentos como prioridade em cada comunidade, em cada região..." 26 (p. 32) e, por conseguinte, também deve ser de responsabilidade dos estados e municípios. Destacam-se também os estados da Bahia e Minas Gerais, que organizaram as metas de seus PlanSAN por diretrizes diferentes daquelas descritas na PNSAN 4. Apesar de o Decreto no 7.272/2010 4 estabelecer as diretrizes consideradas nacionalmente importantes para a garantia de segurança alimentar e nutricional, também orienta que os estados possam adaptar seus planos conforme as especificidades regionais, permitindo planos que se diferenciem, sem prejuízo de sua qualidade, e podendo propiciar um avanço mais significativo na implementação do plano e concretização do SISAN.

Ressalta-se a importância da relação dos PlanSAN com os PPAs dos estados. A organização das ações de governo em PPA que resultem em bens e serviços para a população está previstas na 
Constituição Federal e regulamentada pelo Decreto no 2.829/199827. É o PPA de cada estado que definirá os projetos e ações a serem executados, bem como a distribuição dos recursos financeiros utilizados na gestão do poder executivo. Municípios, estados e o governo federal devem elaborar e executar seus PPAs e, nesse sentido, os planos de políticas públicas específicas, como os PlanSAN, servem para orientar as ações e programas previstos nos PPAs, garantindo que respondam efetivamente pelo DHAA e segurança alimentar e nutricional. Assim, a organização da gestão pública aparece como elemento fundamental para o sucesso da PNSAN 28,29, e os PlanSAN emergem como instrumentos capazes de promover a gestão da PNSAN, organizando a intervenção plurianual do poder público e da sociedade. Ainda que a maioria dos estados com PlanSAN publicados atendam a algumas normativas estabelecidas pela PNSAN, esses instrumentos tornam-se frágeis e pouco exequíveis quando não estão intimamente relacionados aos PPAs, ou apresentam período de vigência diferente dos PPAs. Não estando atrelados aos PPAs, não possuem vinculação orçamentária para suas metas. Com isso, há o risco da existência de planos teoricamente bem estruturados, mas que apresentarão dificuldades para sua execução.

Tão importante quanto construir planos com metas intersetoriais que atendam plenamente ao conceito de segurança alimentar e nutricional, é monitorar a execução dos próprios planos. Quase metade dos planos não apresentou descrição da metodologia de monitoramento ou responsáveis para sua realização. A PNSAN não orienta como esse monitoramento deve ocorrer, mas define que cabe à CAISAN realizar o processo. A CAISAN nacional sugere metodologia de monitoramento mediante PNSAN, complementada com a criação do Sistema de Monitoramento do Plano Nacional de Segurança Alimentar e Nutricional (SISPLANSAN) ${ }^{30}$. Entretanto, esse sistema refere-se à gestão do plano na esfera federal e, consequentemente, necessita de adaptação para os níveis estadual e municipal 31.

A efetividade do plano deve se traduzir na melhoria dos indicadores de segurança alimentar e nutricional e, por isso, a PNSAN determina que os PlanSAN precisam descrever a metodologia para o monitoramento de segurança alimentar e nutricional 4, o que foi uma realidade apenas para os planos da Bahia e Santa Catarina. A definição de indicadores capazes de expressar as dimensões envolvidas no conceito de segurança alimentar e nutricional ainda é um desafio. Propostas de indicadores capazes de avaliar a presença de tais determinantes em nível familiar e territorial foram elaboradas e aplicadas no Brasil. A Escala Brasileira de Insegurança Alimentar (EBIA) aparece como o principal instrumento aplicado no país, sendo utilizada desde 2004 pelo IBGE (http://www.ibge.gov.br/home/ estatistica/populacao/seguranca_alimentar_2004_2009/default.shtm, acessado em 16/Set/2016). em todo o território nacional. Apesar da ampla utilização, sua avaliação centrada na dimensão do acesso aos alimentos e em nível familiar pode ser considerada uma limitação e, em razão disso, outras propostas têm sido apresentadas 32,33 .

O IBGE também vem realizando a Estadic e Pesquisa de Informações Básicas Municipais (Munic), incluindo informações sobre a PNSAN (IBGE. http://www.ibge.com.br/home/estatistica/economia/ estadic/default.shtm, acessado em 01/Nov/2016). Costa et al. 34 demonstraram a possibilidade da combinação de métodos de avaliação de segurança alimentar e nutricional em nível familiar com aspectos territoriais. Em 2014, a CAISAN nacional lançou uma ferramenta de pesquisa anual de informações sobre a gestão do Sistema e da PNSAN nos estados e municípios (MapaSAN) 35. Azevedo \& Ribas 36, considerando as limitações das escalas de mensuração de insegurança alimentar e nutricional em apontar as relações entre a vivência dessa insegurança e os processos e agentes de produção do fenômeno, expõem a necessidade de novos indicadores e metodologias.

O fortalecimento dos planos em nível estadual passa pelo desenvolvimento, por parte do Governo Federal, de estratégias de apoio técnico e financeiro, bem como pela atenção aos processos avaliativos dos planos estaduais 37. Contudo, percebe-se um reduzido número de estudos avaliativos da PNSAN, havendo uma centralidade em estudos sobre programas setoriais de segurança alimentar e nutricional 38,39,40,41. Desse modo, aponta-se a necessidade de avanços teóricos e, particularmente, metodológicos que possam subsidiar a gestão e qualificação da política pública. Acrescenta-se que os incentivos do Governo Federal e o crescente interesse de estados e municípios na implementação dessa política justificam a realização de estudos avaliativos para qualificar este processo. Considerando que os PlanSAN são os instrumentos que sistematizam a programação de ações para promoção de segurança alimentar e nutricional, avaliar sua adequação normativa à PNSAN e sua capacidade de responder por segurança alimentar e nutricional torna-se relevante. 
Dentre os pontos que carecem aprofundamento no âmbito dos PlanSAN, destaca-se o conteúdo das metas, analisando se as mesmas corroboram e dão conta da complexidade do conceito de segurança alimentar e nutricional adotado pelo país. Tal aspecto não foi objeto do presente artigo, no entanto, acredita-se que tal esforço inicial possa facilitar a análise teórica dos planos. Ainda, considera-se fundamental investigar a quantidade de recursos financeiros empregada para o funcionamento da CAISAN e da PlanSAN, com o intuito de melhor compreender os fatores que facilitam e dificultam a consolidação da PNSAN, bem como avaliar sua eficiência. Por fim, reforça-se que a insuficiência de dados secundários atualizados sobre os componentes do SISAN e a divergência de informações entre bases de dados nacionais (Estadic e MapaSAN) apontam para a necessidade de se qualificar o processo de monitoramento de segurança alimentar e nutricional e do SISAN. Porém, reforça-se a percepção de que tais limitações são inerentes ao processo inicial de implementação de uma política pública.

\section{Considerações finais}

Analisaram-se os PlanSAN e se obteve, portanto, um panorama acerca dos aspectos normativos do processo de estruturação do SISAN nos estados brasileiros.

Os planos têm papel fundamental no compromisso assumido pelos governos na execução de ações que respondam pelas demandas emanadas das conferências e do CONSEA. Todos os planos analisados apresentaram inadequações normativas, impondo limites no processo de descentralização do sistema e apontando para a necessidade de qualificação.

Destaca-se entre os achados, o descumprimento nos PlanSAN quanto à adequação de sua vigência aos PPAs estaduais, ou ainda a inexistência de vinculação orçamentária para execução das metas. Tal situação pode implicar menor capacidade de cumprimento dos compromissos assumidos. Cabe refletir sobre a inexistência de um repasse fundo a fundo para a gestão do SISAN, a exemplo de outros sistemas consolidados como Sistema Único de Saúde (SUS) e Sistema Único de Assistência Social (SUAS), que pode justificar a dificuldade de vinculação orçamentária das metas propostas nos planos.

Os resultados demonstram a importância da construção e realização de avaliações dos PlanSAN como forma de garantir sua implementação. Além disso, o fortalecimento das estruturas componentes do SISAN, apoio técnico e formações com CONSEA e, sobretudo com as CAISAN, podem qualificar o processo de construção, monitoramento e avaliação dos planos, sensibilizando atores e permitindo que gestores públicos e sociedade civil apoderem-se desse instrumento.

\section{Colaboradores}

M. L. Machado contribuiu com a concepção da pesquisa, coleta, análise e interpretação dos resultados e redação do manuscrito. C. G. Gabriel e C. Soar contribuíram com a concepção da pesquisa, análise e interpretação dos resultados e redação do manuscrito. G. R. Mamed e M. C. Martins contribuíram com a coleta e análise dos dados e revisão do manuscrito. P. M. O. Machado contribuiu com a análise e interpretação dos resultados e revisão crítica do manuscrito. J. T. Lacerda e M. C. Marcon contribuíram com a concepção da pesquisa e revisão crítica do manuscrito.

\section{Agradecimentos}

Ao Ministério do Desenvolvimento Social (Chamamento Público MDS/SESAN no 01/2013). Às CAISAN nacional e estaduais pela disponibilização de informações. 


\section{Referências}

1. Brasil. Lei no 11.346 , de 15 de setembro de 2006. Cria o Sistema Nacional de Segurança Alimentar e Nutricional - SISAN - com vistas a assegurar o direito humano à alimentação adequada e dá outras providências. Diário Oficial da União 2006; 18 set.

2. Vasconcelos FAG. Combate à fome no Brasil: uma análise histórica de Vargas a Lula. Rev Nutr 2005; 18:439-57.

3. Brasil. Emenda Constitucional no 64, de 4 de fevereiro de 2010. Altera o art. 6o da Constituição Federal, para introduzir a alimentação como direito social. Diário Oficial da União 2010; 4 fev.

4. Brasil. Decreto no 7.272 , de 25 de agosto de 2010. Regulamenta a Lei no 11.346 , de 15 de setembro de 2006, que cria o Sistema Nacional de Segurança Alimentar e Nutricional - SISAN com vistas a assegurar o Direito Humano à Alimentação Adequada, institui a Política Nacional de Segurança Alimentar e Nutricional - PNSAN, estabelece os parâmetros para a elaboração do Plano Nacional de Segurança Alimentar e Nutricional, e dá outras providências. Diário Oficial da União 2010; 26 ago.

5. Burlandy L. A construção da política de segurança alimentar e nutricional no Brasil: estratégias e desafios para a promoção da intersetorialidade no âmbito federal de governo. Ciênc Saúde Coletiva 2009; 14:851-60.

6. Kepple AW, Segall-Corrêa AM. Conceituando e medindo segurança alimentar e nutricional. Ciênc Saúde Coletiva 2011; 16:187-99.

7. Ministério do Desenvolvimento Social e Combate à Fome. Resolução no 3, de 8 de abril de 2014. Torna pública a adesão dos Estados e do Distrito Federal ao Sistema de Segurança Alimentar e Nutricional. Diário Oficial da União 2014; 23 abr.

8. Aliaga MA, Santos SMC. Food and nutrition security public initiatives from a human and socioeconomic development perspective: mapping experiences within the 1996 World Food Summit signatories. Soc Sci Med 2014; 104:74-9.

9. Wiegers ES. The role of the agricultural sector in mitigating the impact of HIV/AIDS in SubSaharan Africa. NJAS - Wageningen Journal of Life Sciences 2008; 56:155-66.

10. Fielden SJ, Anema A, Fergusson P, Muldoon K, Grede N, de Pee S. Measuring food and nutrition security: tools and considerations for use among people living with HIV. AIDS Behav 2014; 18 Suppl 5:S490-504.
11. Malapit HJL, Quisumbing AR. What dimensions of women's empowerment in agriculture matter for nutrition in Ghana? Food Policy $2015 ; 52: 54-63$.

12. Cabral L, Favareto A, Mukwereza L, Amanor K. Brazil's agricultural politics in Africa: more food international and the disputed meanings of "family farming". World Dev 2016; 81:4760.

13. Tirado MC, Crahay P, Mahy L, Zanev C, Neira $\mathrm{M}$, Msangi $\mathrm{S}$, et al. Climate change and nutrition: creating a climate for nutrition security. Food Nutr Bull 2013; 34:533-47.

14. Allen T, Prosperi P, Cogill B, Flichman G. Agricultural biodiversity, social-ecological systems and sustainable diets. Proc Nutr Soc 2014; 73:498-508.

15. Abadie LM, Galarraga I, Milford AB, Gustavsen GW. Using food taxes and subsidies to achieve emission reduction targets in Norway. J Clean Prod 2016; 134:280-97.

16. Allen T, Prosperi P. Modeling sustainable food systems. Environ Manage 2016; 57:956-75.

17. Kesavan PC. Shaping science as the prime mover of sustainable agriculture for food and nutrition security in an era of environmental degradation and climate change. Curr Sci 2015; 109:488-501.

18. Arruda BKG, Arruda IKG. Marcos referenciais da trajetória das políticas de alimentação e nutrição no Brasil. Rev Bras Saúde Matern Infant 2007; 7:319-26.

19. Valente FLS. Fome, desnutrição e cidadania: inclusão social e direitos humanos. Saúde Soc 2003; 12:51-60.

20. Aranha AV, organizador. Fome Zero: uma história brasileira. Brasília: Ministério do Desenvolvimento Social e Combate à Fome; 2010.

21. Bandoni DH, Marchioni DML, Brasil BG, Figueiredo IC, Sarti FM. O programa de incentivo à instalação de cozinhas comunitárias: avaliação de uma política pública brasileira Nutrire Rev Soc Bras Aliment Nutr 2010; 35:15-27.

22. Lima SC, Magalhães R, Fonseca LE, Carvalho A. Segurança Alimentar e Nutricional na comunidade dos países de língua portuguesa: desafios e perspectivas. Rio de Janeiro: World Nutrition; 2012.

23. Balduino $\mathrm{AD}$. Caracterização do processo de construção do I Plano Distrital de Segurança Alimentar e Nutricional - I PDSAN. Brasília: Universidade de Brasília; 2014. 
24 Pinheiro ARO. Reflexões sobre o processo histórico/político de construção da Lei Orgânica de Segurança Alimentar e Nutricional. Segurança Alimentar e Nutricional 2008; 15:1-15.

25. Nascimento AL, Andrade SLLS. Segurança alimentar e nutricional: pressupostos para uma nova cidadania? Ciênc Cult (São Paulo) 2010; 62:34-8.

26. Stedile JP. Soberania alimentar, o que é isso? In: Miyashiro R, organizador. Gastronomia e identidade cultural - coletânea de textos para educandos. Florianópolis: Central Única dos Trabalhadores; 2008. p. 32-4.

27. Brasil. Decreto no 2.829, de 29 de outubro de 1998. Estabelece normas para a elaboração e execução do Plano Plurianual e dos Orçamentos da União, e dá outras providências. Diário Oficial da União 1998; 30 out.

28. Costa CA, Bógus CM. Significados e apropriações da noção de segurança alimentar e nutricional pelo segmento da sociedade civil do Conselho Nacional de Segurança Alimentar e Nutricional. Saúde Soc 2012; 21:103-14.

29. Rocha NC, Doria NG, Boia GM, Bógus CM. Organização e dinâmica do Conselho Municipal de Segurança Alimentar e Nutricional de São Paulo: implicações para a sua atuação na construção da política municipal de Segurança Alimentar e Nutricional. Rev Nutr 2012; 25:133-46.

30. Secretaria de Avaliação e Gestão da Informação, Ministério do Desenvolvimento Social. Sistema de Monitoramento do Plano Nacional de Segurança Alimentar e Nutricional. http:// aplicacoes.mds.gov.br/sagi/plansan/auth.php (acessado em 18/Nov/2016).

31. Machado ML, Machado PMO, Marcon MC, Gabriel CG, Martins MC, Lacerda JT. Avaliação dos planos estaduais de segurança alimentar e nutricional - uma proposta metodológica. Saúde e Transformação Social 2016; 7:1323.

32. Panelli-Martins BE, Santos SMC, Assis AMO. Segurança alimentar e nutricional: desenvolvimento de indicadores e experimentação em um município da Bahia, Brasil. Rev Nutr 2008; 21 Suppl:65s-81s.
33. Conselho Nacional de Segurança Alimentar e Nutricional. A segurança alimentar e nutricional e o direito humano à alimentação adequada no Brasil - indicadores e monitoramento - da Constituição de 1988 aos dias atuais. Brasília: Conselho Nacional de Segurança Alimentar e Nutricional; 2010.

34. Costa LV, Gomes MFM, De Lelis DAS. Food security and agricultural productivity in Brazilian metropolitan regions. Procedia Economics and Finance 2013; 5:202-11.

35. Secretaria de Avaliação e Gestão da Informação, Ministério do Desenvolvimento Social. MapaSAN: mapeamento de SAN nos municípios. http://aplicacoes4.mds.gov.br/sagicenso/ mapasan2015/index.php (acessado em 18/ Nov/2016).

36. Azevedo E, Ribas MTGO. Estamos seguros? Reflexões sobre indicadores de avaliação da segurança alimentar e nutricional. Rev Nutr 2016; 29:241-51.

37. Pereira TST, Heller L. Planos municipais de saneamento básico: avaliação de 18 casos brasileiros. Eng Sanit Ambient 2015; 20:395-404.

38. Santos LMP, Santos SMC, Santana LAA, Henrique FCS, Mazza RPD, Santos LAS, et al. Avaliação de políticas públicas de segurança alimentar e combate à fome no período 19952002. 4 - Programa Nacional de Alimentação Escolar. Cad Saúde Pública 2007; 23:2681-93.

39. Carvalho AT, Almeida ER, Nilson EAF, Ubarana JA, Fernández IM, Immink M. Métodos de análise em programas de segurança alimentar e nutricional: uma experiência no Brasil. Ciênc Saúde Coletiva 2013; 18:309-21.

40. Gabriel CG, Calvo MCM, Vasconcelos FAG, Lacerda JT, Freitas SFT, Schmitz BAS. Avaliação da gestão municipal do Programa Nacional de Alimentação Escolar nos maiores municípios de Santa Catarina. Cad Saúde Pública 2014; 30:2017-23.

41. Gabriel CG, Goulart G, Calvo MCM. Gestão municipal do Programa Nacional de Alimentação Escolar nas capitais da Região Sul do Brasil. Rev Nutr 2015; 28:667-80. 


\section{Abstract}

A descriptive and documental study was performed from August to October 2016 to analyze compliance by state plans for food and nutritional security (PlanSAN) with the guidelines set by the Brazilian National Policy for Food and Nutritional Security (PNSAN). The state plans were accessed via the websites of the Inter-Ministerial Chamber for Food and Nutritional Security (CAISAN) or the state governments, plus complementary data collection at the state level. All the states of Brazil joined the National System for Food and Nutritional Security (SISAN), while fewer than half (13 states, 48\%) had drafted their plans. Of these, 5 (38\%) of the PlanSAN had schedules that coincided with the same state's pluriannual plan, 5 (38\%) of the PlanSAN specified the budget requirements for meeting the proposed targets, 7 (54\%) specified mechanisms for monitoring the plan, and only $2(15 \%)$ defined methodologies for monitoring food and nutritional security. The recent existence of (and adherence to) the CAISAN appear to be related to the lack of state plans in half the states. Although most of the states that did have plans met some of the guidelines laid out by the PNSAN, these mechanisms become weak and impractical when they lack earmarked budget funds to meet their targets. Since the PNSAN is structurally inter-sectorial, the development of plans requires collective work by various government departments. Importantly, the items analyzed here are all guidelines, which implies the need for mechanisms to monitor their actual implementation.

Food and Nutrition Security; Nutrition Programs; Program Evaluation

\section{Resumen}

Con el propósito de analizar la adecuación de los planes estatales de seguridad alimentaria y nutricional (PlanSAN), a las normas establecidas por la Política Nacional de Seguridad Alimentaria y Nutricional (PNSAN), se realizó una investigación descriptiva y documental, con una recogida de datos entre agosto a octubre de 2016. El acceso a los planes se realizó en el sitio web de la Cámara Interministerial de Seguridad Alimentaria y Nutricional (CAISAN) de los gobiernos estatales, con una recogida de información complementaria en el ámbito de los estados. Todos los estados brasileños se adhirieron al Sistema de Seguridad Alimentaria y Nutricional (SISAN), mientras que menos de la mitad (13 estados, un 48\%) elaboró sus planes, destacándose algunos aspectos: $5(38 \%)$ de los PlanSAN tenían una vigencia correspondiente al plan plurianual del estado; 5 (38\%) de los PlanSAN describian los requisitos presupuestarios para la ejecución de las metas propuestas; y 7 (54\%) describian mecanismos de monitoreo del plan y solamente 2 (15\%) definían la metodología para el monitoreo de la seguridad alimentaria y nutricional. El menor tiempo de existencia de la CAISAN $y$ de adhesión parecen estar relacionados con la inexistencia de PlanSAN. A pesar de que la mayoría de los estados con planes atiendan algunas normativas establecidas por la PNSAN, esos instrumentos se convierten en frágiles y poco viables, cuando no poseen una vinculación presupuestaria para sus metas. Debido al hecho de que el PNSAN sea estructuralmente intersectorial, la construcción de los planes depende de un trabajo colectivo de las diversas secretarias de gobierno. Se refuerza que los items analizados fueron todos normativos, lo que implica necesidad del estabelecimiento de mecanismos que garanticen su adecuada ejecución.

Seguridad Alimentaria y Nutricional; Programas de Nutrición; Evaluación de Programas y Proyectos de Salud
Recebido em 05/Dez/2016

Versão final reapresentada em 18/Abr/2017 Aprovado em 25/Abr/2017 